\title{
Fusarium napiforme systemic infection: case report with molecular characterization and antifungal susceptibility tests
}

\author{
Marcela de Souza ${ }^{1,4^{*}}$, Tetsuhiro Matsuzawa², Luzia Lyra ${ }^{3}$, Ariane Fidelis Busso-Lopes ${ }^{1}$, Tohru Gonoi²,
} Angélica Zaninele Schreiber ${ }^{3}$, Katsuhiko Kamei ${ }^{2}$, Maria Luiza Moretti ${ }^{1}$ and Plínio Trabasso ${ }^{1}$

\begin{abstract}
Introduction: During the last decades, Fusarium spp. has been reported as a significant cause of disease in humans, especially in immunocompromised patients, who have high risk of invasive life-threatening disease. Fusarium species usually reported as cause of human disease are F. solani, F. oxysporum and F. verticillioides.

Case description: We describe the second case in the literature of disseminated fusariosis caused by Fusarium napiforme, that occurred in a 60-year-old woman with multiple myeloma after subsequent cycles of chemotherapy.

Discussion and Evaluation: We identified the F. napiforme not only by standard morphologic criteria by macroscopic and microscopic characteristics, but also confirmed by molecular biology methods, including sequencing. The antifungal susceptibility of the $F$. napiforme isolates were tested to seven antifungal drugs; the azoles were the most active drug against all the isolates tested.

Conclusions: Fusarium spp. are of relevance in medical mycology, and their profiles of low susceptibility to antifungal drugs highlight the importance for faster and more accurate diagnostic tests, what can contribute to an earlier and precise diagnosis and treatment.
\end{abstract}

Keywords: Fusariosis; Opportunistic Infections; Immunocompromised host

\section{Background}

Fusarium are widely distributed fungi in soil, plants, plant debris and other organic substrates, and in water systems. During the last decades, Fusarium spp. has been reported as a significant cause of disease in humans, especially immunocompromised patients (Jureen et al. 2008; Bourgeois et al. 2010; De Pinho et al. 2012; Calcaterra et al. 2013).

In immunocompetent persons, Fusarium usually causes localized infections (Bourgeois et al. 2010; De Pinho et al. 2012; Calcaterra et al. 2013; Homa et al. 2013). Conversely, immunocompromised hosts, mainly those with acute onco-hematological diseases or after allogeneic hematopoietic stem cell transplant, have high risk of invasive life-threatening diseases. In such patients, invasive

\footnotetext{
* Correspondence: marceladesouza88@gmail.com

'Department of Internal Medicine, School of Medicine, University of Campinas, Campinas, São Paulo, Brazil

${ }^{4}$ LIM 46 - Laboratory of Parasitology - HC/FMUSP, São Paulo, Brazil Full list of author information is available at the end of the article
}

fusariosis (IF) is relatively resistant to standard antifungal therapy limiting their treatment options (Scheel et al. 2013; Pereira et al. 2013).

Fusarium species reported as cause of human disease and $F$. solani, $F$ oxysporum and $F$. verticillioides are the most frequently species causing IF (Gupta et al. 2000; Tezcan et al. 2009).

The first case of disseminated fusariosis was described in 1973 (Cho et al. 1973). Since then, there was a significant increase in the occurrence of disseminated disease, probably reflecting the increase in number of immunocompromised hosts (Bourgeois et al. 2010). There are reports of IF worldwide (Martino et al. 1994; Nucci and Anaissie 2007; Tortorano et al. 2008; Slavin et al. 2012; Nucci et al. 2013). The first case of disseminated fusariosis due to F. napiforme was described in 1993 (Melcher et al. 1993) but, there were no subsequent reports of disseminated disease. Thus, to our knowledge, we describe the second case of the literature of disseminated fusariosis caused by 
F.napiforme, and the first report of F. napiforme confirmed by molecular biology methods, including sequencing.

\section{Case description and molecular identification of the clinical isolates \\ Case description}

A 60-year-old woman was diagnosed with stage IIIB multiple myeloma (MM) in 2005. She was treated with chemotherapy, 6 cycles of VAD (vincristine + doxorubicin + dexamethasone) in 2006, then cyclophosphamide in 2007. This treatment was followed by an autologous bone marrow transplant in 2008. The disease relapsed in October 2010. She underwent decompressing lumbar spine surgery in 2010 and received various courses of treatment with zoledronic acid throughout her followup. The patient had also radiotherapy in 2009 (spine) and 2011 (spleen), followed by salvage chemotherapy throughout 2011 (cyclophosphamide + thalidomide + dexamethasone-CTD, then velcade + thalidomide + dexamethasone-VTD, and finally cyclophosphamide + prednisone). At this point she was considered to present a very good partial response (VGPR). In October 2012, during a routine medical consultation, she complained of palpitation and dyspnea starting 10 days before. The patient presented with hypercalcemia and had to be admitted for the treatment of this condition. During the admission, a second BMT was proposed to treat the refractory MM; therefore, myelosuppressive chemotherapy was initiated. After one week the neutrophil count fell to zero and painful vasculitis lesions arose in lower abdominal region and in the left thigh. Blood cultures were withdrawn and skin biopsy was performed. Antimicrobial therapy was started with cefepime and amphotericin B deoxycholate. Blood culture resulted positive for Pseudomonas aeruginosa and cefepime was switched to imipenem. After one week of this regimen, patient was still neutropenic, febrile and worsening clinical conditions. A new set of blood cultures was positive for $\mathrm{Fu}$ sarium sp. The skin biopsy showed a large number of hyphae using direct microscopic exam, morphologically compatible with fusariosis. The patient died in November due to refractory septic shock.

\section{Fusarium samples}

Four clinical isolates of Fusarium sp. from clinical specimens were identified as: LIF 2008, 2009 and 2010 recovered from blood cultures (BacT/ALERT ${ }^{\circ}$ 3D, bioMérieux AS, France), LIF 1994 from skin biopsy and F111 from the air of the patient's hospital room.

\section{Microorganism identification}

The five clinical isolates were cultured in Sabouraud Dextrose agar (Difco, Sparks, Maryland, USA) and identified by morphologic criteria after subculture by macroscopic and microscopic characteristics (Verweij et al. 2007).

\section{Molecular methods}

DNA extraction of Fusarium spp. from blood culture bottles, from skin biopsy and from air were performed using Dr. GenTLE ${ }^{\circ}$ kit (Takara, Otusu, Shiga, Japan). After DNA measurement in NanoDrop 2000 (Thermo Scientific, Wilmington, USA) and equalization to a concentration of $2 \mathrm{ng} / \mu \mathrm{L}$, DNA samples were analyzed using: DNA microarray and DNA sequencing.

The DNA microarray was performed as described by (Ferrari et al. 2013), the oligonucleotide probes, consisting of 14 to 20 species-specific nucleotide sequences with biotin-labeled poly $\mathrm{T}$ anchors at the end of each nucleotide (Invitrogen, Showajima, Japan), were designed based on ITS1 and ITS2 sequences of the Type strains [GenBank database, American Type Culture Collection (ATCC), Centraalbureau voor Schimmelcultures (CBS) and MMRC-Chiba (IFM)]. Multiple-sequence alignments were performed using the BioEdit software (version 7.1.3. [http://www.mbio.ncsu.edu/BioEdit/bioedit.html]). Conserved regions were also used as targets for genus-specific probes or as controls. The probe sequences were spotted onto a plastic slide (NGK Insulators LTD, Aichi, Japan) using a KCS mini microarray printer (Kubota Comps. Corporation, Amagasaki, Japan). For fungal identification, PCR using universal fungal primers ITS1 (5' -TCCGTAGGTG AACCTGCGG-3') and ITS4 (5' ${ }^{\prime}$-TCCTCCGCTTATTGA TATGC-3') (Sigma-Aldrich, Saint Louis, USA) were used to amplify the ITS regions (ITS1 and ITS2) and the 5.8S rRNA gene followed by hybridization, conjugation, coloration and direct visualization of specifically positioned spots on the slide that were consisted of 23 fungal species.

The sequencing was performed as described before (Moreira-Oliveira et al. 2005), with modifications. First, we performed a polymerase chain reaction (PCR) to amplify a region of the gene EF1 $\alpha$ using the pairs of primers forward HS392 (5'-TCAAAATGGGTAAGGA (A/G)GACAAGAC-3') and HS393 (5'-GCCTGGGA(G/ A)GTACCAGT(G/C)ATCATGTT-3'). The PCR products were directly sequenced with a BigDye $\odot$ terminator reagent kit (Applied Biosystems, Foster City, CA) in an automated DNA sequencer (3110 AB System, Applied Biosystems, Foster City, USA). Besides HS392 and HS393 primers, two internal initiators were used for sequencing reaction and amplification of EF1 $\alpha$ gene: EF11 (5'-GTGG GGCATTTACCCCGCC-3') and EF21 (5'-GAGTGGCG GGGTAAATGCC-3'). The sequences were assembled using ATSQ version 6.0.1 (Genetix) and compared with database information available at NCBI bank (National Center for Biotechnology Information, http://blast.ncbi. nlm.nih.gov/Blast.cgi). Sequences alignment was carried 


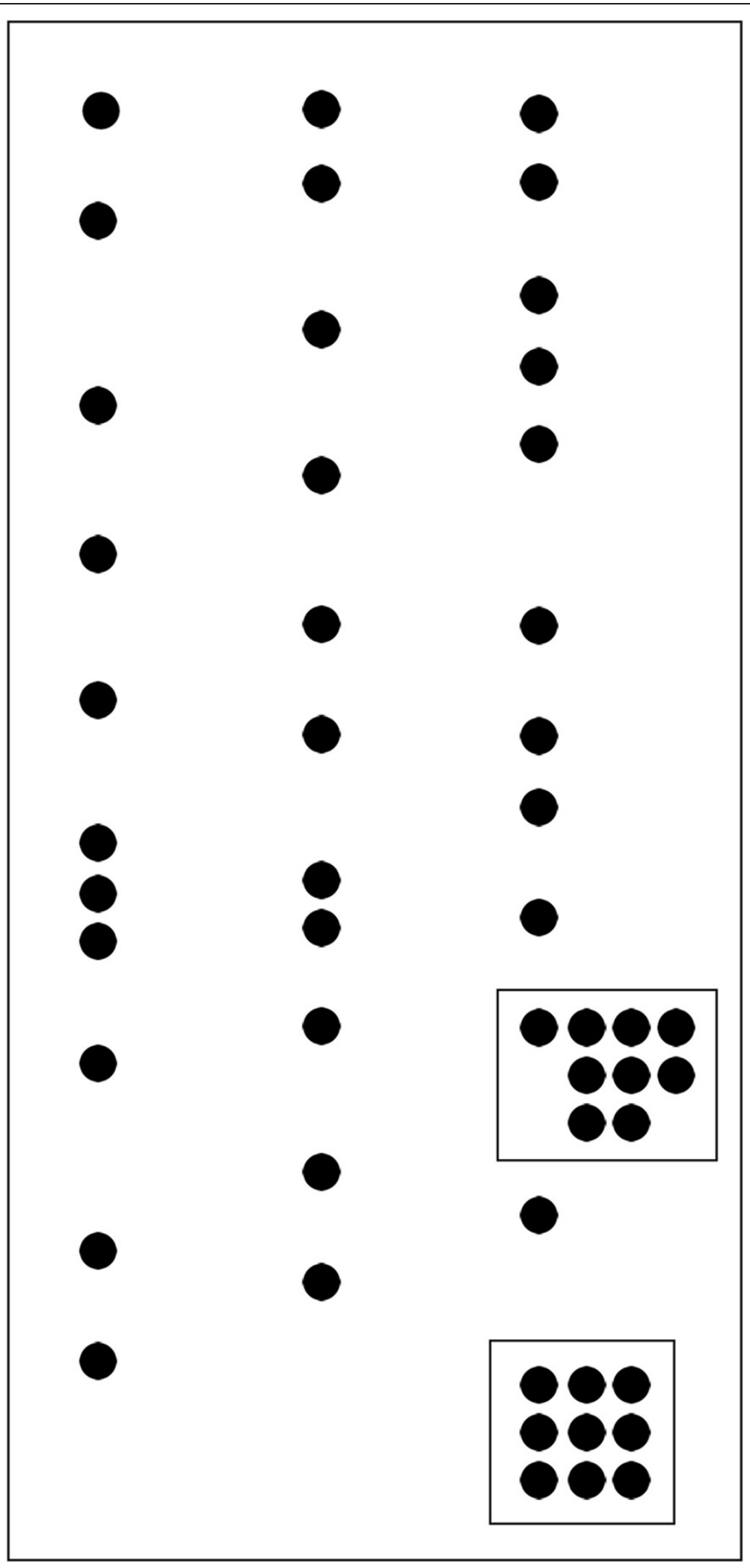

Figure 1 Representative hybridization pattern of Fusarium non-solani species complex and positive control using DNA microarray. A group of specific hybridization spots are visualized inside the square above and the spots remaining are representative of biotin (negative for others species). The positive control is shown inside the below square and represents a sequence common to all fungal species. 
out in Clustal Omega (http://www.ebi.ac.uk/Tools/msa/ clustalo/). The sequencing was performed in the Molecular Epidemiology Laboratory at the Faculty of Medical Sciences, State University of Campinas, São Paulo, Brazil and the Medical Mycology Research Center, Chiba University, Chiba, Japan.

\section{Antifungal susceptibility test}

Antifungal susceptibility tests were performed for samples LIF 2008, LIF 2009, LIF 2010, 1994 and EF111. Minimal inhibitory Concentrations (MIC) and minimal effective concentration (MEC) were determined following the micro dilution method recommended by CLSI document M38A2, with minor changes. The conidia forms were suspended by gently probing the colony with a sterile Pasteur pipette tip to dislodge the conidia from the hyphal mat and the solution; then, they were counted in a Neubauer chamber and adjusted to a suspension containing $4 \times 10^{4}$ conidia/mL (CLSI 2008; Teixeira et al. 2005). After that, conidia were re-suspended in RPMI 1640 (Sigma) with Lglutamine, without sodium bicarbonate, and buffered with $0.165 \mathrm{~mol} / \mathrm{L}$ 3-morpholinopropanesulfonic acid (MOPS) in $\mathrm{pH}$ 7.0. The final suspension was distributed in microdilution plates containing pre-defined incremental concentrations of amphotericin B (AMB); itraconazole (ITZ); voriconazole (VOR); micafungin (MCF); 5-flucytosine (5FC); miconazole (MCZ) and fluconazole (FCZ). The plates were incubated at $35^{\circ} \mathrm{C}$ and fungal growth was eye-observed after $24 \mathrm{~h}$ and $48 \mathrm{~h}$. The MIC was defined as the lowest drug concentration that caused $100 \%$ of inhibition of visible fungal growth for AMB, ITZ and VCZ and as the lowest drug concentration that caused $50 \%$ of inhibition of visible fungal growth for MCZ, 5FC and FCZ and the MEC for MCZ was defined as the lowest concentration of drug that leads to the growth of small, rounded, compact hyphal forms as compared to the hyphal growth seen in the growth control well. The tests were performed in duplicate. American Type Culture Collection strains Candida parapsilosis ATCC 22019, Candida krusei ATCC 6258, Candida albicans ATCC 76615 and Candida albicans ATCC 90028 were used for quality control.

\section{Antifungal agents}

Micafungin, amphotericin B, 5-flucytosine, fluconazole, voriconazole, itraconazole and miconazole in preprepared dry plates (Eiken Chemical Co., Ltd., Tokyo) (Makimura et al. 2005).

\section{Results}

Assessment of the Fusarium napiforme strains

All isolates from blood, skin biopsy and from air were identified as Fusarium sp. by macroscopic and microscopic characteristics, as Fusarium non-solani species complex (FNSSC) DNA microarray methodology (Figure 1) and as F. napiforme (non-solani) by sequencing (Table 1). The sequences alignment showed two F. napiforme distinct groups based on DNA homology: one group with $F$. napiforme samples isolated from blood $(2009,2010)$ and from skin biopsy (1994), and another containing isolates for blood (2008) and air (F111), as shown in Figure 2.

The susceptibility results can be seen in Table 2 .

\section{Discussion}

Fusarium spp. are emerging as pathogens that can cause life-threatening invasive opportunistic infections, mainly among patients with bone marrow suppression and neutropenia. Currently, Fusarium spp. are considered the second most-common mold as cause of opportunistic infection in these patients, being Aspergillus spp. the first ones (Bodey et al. 2002; Cooke et al. 2009). Fusarium spp. are also the most common cause of fungemia with skin manifestations. (Bodey et al. 2002) reported $76 \%$ of 46 patients with hematologic malignancies or solid tumors, considered to have definite Fusarium infections, had skin lesions. (Nucci and Anaissie 2007) also reported 61 hematopoietic stem cell transplant recipients with hematologic malignancies or solid tumors with disseminated Fusarium infection, and metastatic skin lesions was the most frequent clinical presentation, occurring in $46(75 \%)$ of patients.

The combination of cutaneous lesion and positive blood cultures, involving or not other sites are the most frequent pattern of disseminated fusariosis. The most common clinical presentation is a persistently febrile

Table 1 Results of identification of Fusarium species according macro- and micro- morphological characteristics, DNA microarray and DNA sequencing

\begin{tabular}{|c|c|c|c|c|}
\hline Isolate LIF & Macro- and micro-morphological characteristics & DNA microarray & DNA sequencing & $\%$ Similarity* \\
\hline 2008 & Fusarium sp. & FNSSC & Fusarium napiforme & 98 \\
\hline 2009 & Fusarium sp. & FNSSC & Fusarium napiforme & 99 \\
\hline 2010 & Fusarium sp. & FNSSC & Fusarium napiforme & 99 \\
\hline 1994 & Fusarium sp. & FNSSC & Fusarium napiforme & 99 \\
\hline F111 & Fusarium sp. & FNSSC & Fusarium napiforme & 98 \\
\hline
\end{tabular}

LIF = Fungal Research Laboratory, FNSSC = Fusarium non-solani species complex, ${ }^{*}$ Similarity $\%$ for the comparison with NCBI database. 
2009_Fusarium_napiforme_blood 2010 Fusarium napiforme blood 1994 Fusarium napiforme skin F111_Fusarium_napiforme_air 2008_Fusarium_napiforme_blood

2009 Fusarium napiforme blood 2010 Fusarium napiforme blood 1994_Fusarium_napiforme_skin F111_Fusarium_napiforme_air 2008_Fusarium_napiforme_blood

2009 Fusarium napiforme blood $2010^{-}$Fusarium napiforme blood 1994_Fusarium_napiforme_skin F111_Fusarium_napiforme_air 2008_Fusarium_napiforme_blood

2009 Fusarium napiforme blood 2010_Fusarium_napiforme_blood 1994_Fusarium_napiforme_skin F111_Fusarium_napiforme_air 2008_Fusarium_napiforme_blood

2009_Fusarium_napiforme_blood 2010_Fusarium_napiforme_blood 1994 Fusarium_napiforme_skin F111 Fusarium napiforme air 2008_Fusarium_napiforme_blood

2009_Fusarium napiforme_blood 2010_Fusarium_napiforme_blood 1994_Fusarium napiforme skin F111 Fusarium napiforme air 2008_Fusarium_napiforme_blood

2009_Fusarium_napiforme_blood 2010_Fusarium napiforme_blood 1994_Fusarium napiforme_skin F111 Fusarium napiforme air 2008_Fusarium_napiforme_blood

2009_Fusarium_napiforme_blood 2010_Fusarium_napiforme_blood 1994 Fusarium napiforme skin F111_Fusarium_napiforme_air 2008_Fusarium_napiforme_blood

2009_Fusarium napiforme blood 2010 Fusarium napiforme blood 1994 Fusarium napiforme skin F111_Fusarium_napiforme_air 2008_Fusarium_napiforme_blood

2009 Fusarium napiforme blood 2010 Fusarium napiforme blood 1994_Fusarium_napiforme_skin F111_Fusarium_napiforme_air 2008_Fusarium_napiforme_blood

2009 Fusarium napiforme blood 2010_Fusarium_napiforme_blood 1994_Fusarium_napiforme_skin F111_Fusarium_napiforme_air 2008_Fusarium_napiforme_blood

2009 Fusarium napiforme blood 2010_Fusarium_napiforme_blood 1994_Fusarium_napiforme_skin F111_Fusarium napiforme air

2008_Fusarium_napiforme_blood

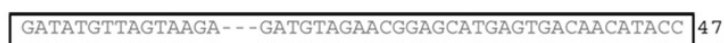
GATATGTTAGTAAGA ----GATGTAGAACGGAGCATGAGTGACAACATACC 47 GATATGTTAGTAAGA----GATGTAGAACGGAGCATGAGTGACAACATACC 47 GGCATGTTAGTAAGAAGAGATGTAGTACGGATCA-GAGCGACAACATACC 49 GGCATGTTAGTAAGAAGAGATGTAGTACGGATCA-GAGCGACAACATACC 49

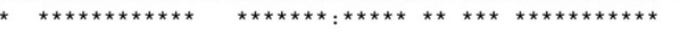

AATGACGGTGACATAGTAGCGAGGAGTCTCGAACTTCCAGAGAGCAATAT 97 AATGACGGTGACATAGTAGCGAGGAGTCTCGAACTTCCAGAGAGCAATAT 97 AATGACGGTGACATAGTAGCGAGGAGTCTCGAACTTCCAGAGAGCAATAT 97 AATGACGGTGACATAGTAGCGAGGAGTCTCGAACTTCCAGAGAGCAATAT 99 AATGACGGTGACATAGTAGCGAGGAGTCTCGAACTTCCAGAGAGCAATAT 99 $* * * * * * * * * * * * * * * * * * * * * * * * * * * * * * * * * * * * * * * * * * * * * * * * * * * * * * *$

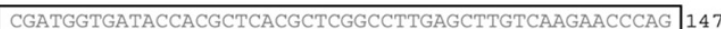
CGATGGTGATACCACGCTCACGCTCGGCCTTGAGCTTGTCAAGAACCCAG 147 CGATGGTGATACCACGCTCACGCTCGGCCTTGAGCTTGTCAAGAACCCAG 147 CGATGGTGATACCACGCTCACGCTCGGCCTTGAGCTTGTCAAGAACCCAG 149 CGATGGTGATACCACGCTCACGCTCGGCCTTGAGCTTGTCAAGAACCCAG 149

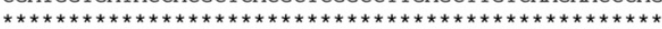

GCGTACTTGAAAGAACCCTTACCGAGCTCAGCGGCTTCCTATTGTCGGAT 197 GCGTACTTGAAAGAACCCTTACCGAGCTCAGCGGCTTCCTATTGTCGGAT 197 GCGTACTTGAAAGAACCCTTACCGAGCTCAGCGGCTTCCTATTGTCGGAT 197 GCGTACTTGAAGGAACCCTTACCGAGCTCAGCGGCTTCCTATTGTCGGAT 199 GCGTACTTGAAGGAACCCTTACCGAGCTCAGCGGCTTCCTATTGTCGGAT 199

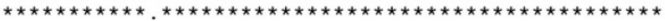

GGTTAGTGACTGCTTGACACGTGACGATGCGCTCAGTGAGGTTGTGGAAT 247 GGTTAGTGACTGCTTGACACGTGACGATGCGCTCAGTGAGGTTGTGGAAT 247 GGTTAGTGACTGCTTGACACGTGACGATGCGCTCAGTGAGGTTGTGGAAT 247 GGTTAGTGACTGCTTGACACGTGACGATGCGCTCAGTGAGGTTGTGGAAT 249 GGTTAGTGACTGCTTGACACGTGACGATGCGCTCAGTGAGGTTGTGGAAT 249

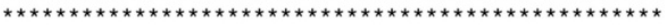

GAGAGAGGGCAGAAACGCGCCGCTCGAGTGGCGGGGTAAATGCCCCACCA 297

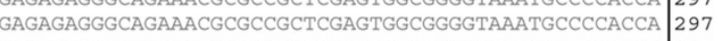
GAGAGAGGGCAGAAACGCGCCGCTCGAGTGGCGGGGTAAATGCCCCACCA 297 GAGAGAGGGCAGAAACGCGCCGCTCGAGTGGCGGGGTAAATGCCCCACCA 299 GAGAGAGGGCAGAAACGCGCCGCTCGAGTGGCGGGGTAAATGCCCCACCA 299

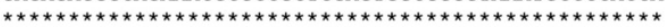

AAAAAATTACGGTCATATCGCAAAATTTTTGAACTCAAGCGGGTAGTGG 347 AAAAAATTACGGTCATATCGCAAAATTTTTGAACTCAAGCGGGGTAGTGG 347 AAAAAATTACGGTCATATCGCAAAATTTTTGAACTCAAGCGGGGTAGTGG 347 AAAAAATTACGGTCATATCGCAAAATCTTTGAACTCAAGCGGGGTAGTGG 349 AAAAAATTACGGTCATATCGCAAAATCTTTGAACTCAAGCGGGGTAGTGG 349

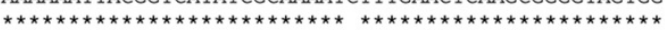

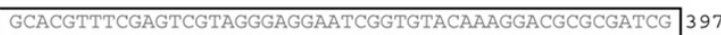
GCACGTTTCGAGTCGTAGGGAGGAATCGGTGTACAAAGGACGCGCGATCG 397 GCACGTTTCGAGTCGTAGGGAGGAATCGGTGTACAAAGGACGCGCGATCG 397 GCACGTTTCGAGTCGTAGGGAGGAATCGGTGTACAAAGGACGCGCGATCG 399 GCACGTTTCGAGTCGTAGGGAGGATCGGTGTACAAAGGACGCGCGATCG 399

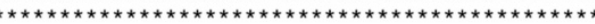

AAGGGAATGTGACTAACCTTCTCGAACTTCTCGATGGTTCGCTTGTCGAT 447 AAGGGAATGTGACTAACCTTCTCGAACTTCTCGATGGTTCGCTTGTCGAT 447 AAGGGAATGTGACTAACCTTCTCGAACTTCTCGATGGTTCGCTTGTCGAT 447 AAGGGAATGTGACTAACCTTCTCGAACTTCTCGATGGTTCGCTTGTCGAT 449 AAGGGAATGTGACTAACCTTCTCGAACTTCTCGATGGTTCGCTTGTCGAT 449

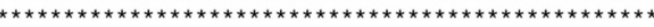

ACCACCGCACTGGTAGATCAAGTGACCGGTCTGTGAAGCGATGTCAGCAT 497 ACCACCGCACTGGTAGATCAAGTGACCGGTCTGTGAAGCGATGTCAGCAT 497 ACCACCGCACTGGTAGATCAAGTGACCGGTCTGTGAAGCGATGTCAGCAT 497 \begin{tabular}{ll}
4 & \\
ACCACCGCACTGGTAGATCAAGTGACCGGTCTGTGAAGCGATGTCAGCAT & 497 \\
\hline ACCACCCACTGGTAGATCAAGTGACCGGTCTGTGAAGCGATGTCAGCAT & 499
\end{tabular} ACCACCGCACTGGTAGATCAAGTGACCGGTCTGTGAAGCGATGTCAGCAT 499

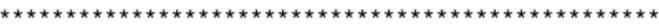

GTTTTCTTTGAGAGATACCCCGCCAGGTCTTGGTCAGGCTTACGATGGCC 547

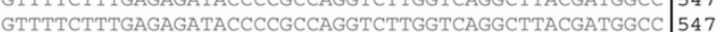
\begin{tabular}{l|l} 
& \\
GTTTTCTTTGAGAGATACCCCGCCAGGTCTTGGTCAGGCTTACGATGGCC & 547
\end{tabular} GTTTTCTTTGAGAAATATCCCGCCATGTCTTGGTCGGGTTTACGATGGCC 549 GTTTTCTTTGAGAAATATCCCGCCATGTCTTGGTCGGGTTTACGATGGCC 549

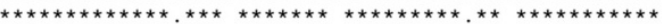

GATAAGCTCATCGTCAAGGGTAGTACTCACAGTGGTCGACTT 589 GATAAGCTCATCGTCAAGGGTAGTACTCACAGTGGTCGACTT 589 GATAAGCTCATCCTCAAGGGIAGTACTCACAGIGGTCGACTT 589 GATAAGCTCATCGTCAAGGGTAGTACTCACAGTGGTCGACTT 591 GATAAGCTCATCGTCAAGGGTAGTACTCACAGTGGTCGACTT 591

Figure 2 Clustal Omega multiple sequences alignment of Fusarium napiforme isolates by EF1a gene sequencing. The * symbol indicate a $100 \%$ homology for the specific base position. The square indicates one of the genetic variant groups. 
Table 2 Literature review of antifungal drugs evaluated for Fusarium species

\begin{tabular}{|c|c|c|c|c|c|c|c|c|}
\hline \multirow[b]{2}{*}{ Author [reference] } & \multirow[b]{2}{*}{ Fusarium species } & \multicolumn{7}{|c|}{ Antifungal agent $\mu \mathrm{g} / \mathrm{ml}(\mathrm{MEC} / \mathrm{MIC})^{*}$} \\
\hline & & AMB & FLU & MCF & ITZ & VOR & $5 \mathrm{FC}$ & MCZ \\
\hline (Melcher et al. 1993) & Fusarium napiforme & 1.16 & 10 & & 1.25 & & $>322.7$ & \\
\hline (Durand-Joly et al. 2003) & Fusarium solani & 1 & & & & & & \\
\hline (Rodriguez et al. 2003 ) & Fusarium oxysporum & 2 & & & & 4 & & \\
\hline (Rothe et al. 2004) & Fusarium oxysporum & 2 & $>64$ & & $<2$ & & $>64$ & \\
\hline (Guzman-Cottrill et al. 2004) & Fusarium solani & 8 & $>64$ & & & 8 & $>64$ & \\
\hline (Teixeira et al. 2005) & Fusarium solani & 2 & & & 128 & & & \\
\hline (Ho et al. 2007) & Fusarium solani & 1 & & & $>16$ & 4 & & \\
\hline (Neuburger et al. 2008) & Fusarium proliferatum & $4-8$ & $>16$ & & $>16$ & 8 & & \\
\hline \multirow[t]{5}{*}{ (Tortorano et al. 2008) } & Fusarium verticillioides & 1.53 & & & 3.33 & 1.74 & & \\
\hline & Fusarium solani & 1.25 & & & $>16$ & 9.21 & & \\
\hline & Fusarium proliferatum & 1.7 & & & $>16$ & 4.2 & & \\
\hline & Fusarium oxysporum & 2.3 & & & $>16$ & 4 & & \\
\hline & Fusarium subglutinans & 3.3 & & & 10.8 & 5.6 & & \\
\hline (Xie et al. 2008) & Fusarium solani & & & & & & & 8 \\
\hline (Tezcan et al. 2009) & Fusarium verticillioides & $1-2$ & & & $>8$ & 4 & & \\
\hline (Bose et al. 2011) & Fusarium spp. & $2-4$ & & & & & & \\
\hline (Liu et al. 2011) & Fusarium solani & 1 & & & & 4 & & \\
\hline (Sekeroglu et al. 2012) & Fusarium solani & 0,5 & $>64$ & & & 8 & & \\
\hline \multirow[t]{2}{*}{ (Pereira et al. 2013) } & Fusarium solani & $>8$ & & & $>8$ & $0.5-0.128$ & & \\
\hline & Fusarium oxysporum & $\leq 2$ & & & 8 & & & \\
\hline (Fanci et al. 2013) & Fusarium verticillioides & 2 & & & & 8 & & \\
\hline \multirow[t]{3}{*}{ (Inano et al. 2013) } & Fusarium solani & & $>4$ & $>16$ & $>8$ & 8 & & 16 \\
\hline & Fusarium moniliforme & & & & & & & 8 \\
\hline & Fusarium oxysporum & & & & & & & 8 \\
\hline \multicolumn{9}{|l|}{ Present study } \\
\hline isolate 2008 & Fusarium napiforme & $2-4$ & $1-2$ & $>16$ & $>8$ & $>8$ & $>64$ & $0.125-0.25$ \\
\hline isolate 2009 & Fusarium napiforme & $2-4$ & $1-2$ & $>16$ & $>8$ & 2 & $>64$ & $0.125-0.25$ \\
\hline isolate 2010 & Fusarium napiforme & $2-4$ & $1-2$ & $>16$ & $>8$ & 4 & $>64$ & $0.125-0.25$ \\
\hline isolate 1994 & Fusarium napiforme & 2 & $1-2$ & $>16$ & $>8$ & 4 & $>64$ & 0.125 \\
\hline isolate F111 & Fusarium napiforme & 1 & 8 & $>16$ & $>8$ & 2 & $>64$ & 0.5 \\
\hline
\end{tabular}

*MEC = minimal effective concentration, $\mathrm{MIC}=$ minimum inhibitory concentration, $\mathrm{MEC}$ was defined for micafungin and MIC for the other drugs, $\mathrm{AMB}=$ amphotericin $\mathrm{B}$, $\mathrm{FLU}=$ fluconazole, $\mathrm{MCF}=$ micafungin, $\mathrm{ITZ}=$ itraconazole, $\mathrm{VOR}=$ voriconazole, $5 \mathrm{FC}=5$-flucytosine, $\mathrm{MCZ}=$ miconazole.

patient with prolonged and profound neutropenia who develops disseminated characteristic skin lesions, with a positive blood culture for a filamentous fungi (Nucci and Anaissie 2007). This was the case of our patient.

The most common Fusarium involved in human infections are F.solani, F.oxysporum and F.verticillioides (Gupta et al. 2000; Tezcan et al. 2009). To our knowledge, we describe here the second case of disseminated fusariosis caused by F.napiforme, and the first case with identification confirmed by molecular techniques, including sequencing.

The first invasive case by F.napiforme was described in 1993 (Melcher et al. 1993) in a patient diagnosed with acute myeloid leukemia, under cytoreduction and profound granulocytopenia following high-dose of cytosine arabinoside, mitoxantrone, and VP-16. Since then, no other cases have been described.

The diagnosis of Fusarium in laboratory includes some criteria such a positive direct mycological examination showing typical septated hyphae branching at $45^{\circ}$. However, the identification of Fusarium to the species level is often difficult and requires a specialized laboratory and skilled personnel. In such situations, molecular biology techniques might be helpful for the definitive diagnosis. Furthermore, the early diagnosis of invasive disease might be helpful to guide the correct antifungal therapy, 
which is crucial for patient recovery (Galimberti et al. 2012; Busemann et al. 2009; Azor et al. 2009). In our study, molecular methods allowed a faster and accurate identification of the causative agent as belonging to F.napiforme group. In addition, the strains F111 and 2008 , from blood and air respectively, showed $100 \%$ sequencing alignment, suggesting that the the air may have been the source of the infection. Samples 2009 and 2010, isolated from the blood, and skin isolate 1994 were also aligned. Thus, it seems that we had two variants of F.napiforme. Therapy for invasive fusariosis is a challenging problem, mainly because Fusarium shows high MICs to antifungal agents, and therefore, there is no proven effective treatment regimen (Tezcan et al. 2009; Guzman-Cottrill et al. 2004; Rothe et al. 2004). The treatment of choice for invasive fusariosis is amphotericin B. However, it is controversial, since there are reports of Fusarium showing MICs for AMB ranging from 1 to $4 \mu \mathrm{g} / \mathrm{mL}$. Triazoles, as voriconazole and posaconazole, have also been used successfully (Pereira et al. 2013; Tortorano et al. 2008). Furthermore, different Fusarium can exhibit variable susceptibility patterns.

In the present case, patient was treated with amphotericin B deoxicolate, initiated after laboratory confirmation of Fusarium fungemia. However, the time between the onset of symptoms and the blood culture was fifteen days and the time between the blood culture and the positive result for Fusarium was fifteen days more. The isolate of F.napiforme in the present case was resistant to amphotericin B, with MIC ranging from $2-4 \mu \mathrm{g} / \mathrm{ml}$. Delay in antifungal therapy plus the resistance profile could have contributed to the patient's death three days after antifungal drug was initiated.

In conclusion, Fusarium spp. are emerging as a fungi of relevance in medical mycology, since they are associated with low susceptibility profiles to antifungal drugs and high mortality rate, mainly in imunocompromissed patients. These facts highlight the importance for faster and more accurate diagnostic tests, contributing to earlier and precise diagnosis and treatment of this lifethreatening infection.

\section{Nucleotide sequence accession numbers}

The sequences determined in this study have been submitted in (Sakai et al. 2014) and deposited in NCBI database with the accession numbers KM099396 to KM099400.

\section{Consent}

Informed consent was obtained from the patient for the publication of this report and any accompanying images.

\section{Competing interests}

The authors report no conflict of interest concerning the materials or methods used in this study or the findings specified in this paper.

\section{Authors' contributions}

MS carried out all the molecular techniques, the sequence alignment and drafted the manuscript. TM carried out the LAMP technique and revised the manuscript. LL carried out the drug susceptibility tests and revised the manuscript. AFBL carried out the DNA microarray technique, the sequence alignment and revised the manuscript. TG is a chief adviser of the research and revised the manuscript. AZS is a chief adviser the research, carried out the drug susceptibility tests and revised the manuscript. KK is a chief adviser of the research and revised the manuscript. MLM is a chief advisor of the research and revised the manuscript. PT is a chief advisor of the research, carried out the LAMP and DNA microarray techniques and drafted the manuscript. All authors read and approved the final manuscript.

\section{Acknowledgements}

This work was supported by JST/JICA, and SATREPS, through the Cooperation Research Grant 02P-29548-09. Marcela de Souza have received a master degree scholarship from the São Paulo Research Foundation (FAPESP), grant number FAPESP 2011/16205-5.

\section{Author details}

${ }^{1}$ Department of Internal Medicine, School of Medicine, University of Campinas, Campinas, São Paulo, Brazil. ${ }^{2}$ Medical Mycology Research Center, Chiba University, Chiba, Japan. ${ }^{3}$ Department of Clinical Pathology, School of Medicine, University of Campinas, Campinas, São Paulo, Brazil. ${ }^{4}$ LIM 46 Laboratory of Parasitology - HC/FMUSP, São Paulo, Brazil.

Received: 29 July 2014 Accepted: 27 August 2014

Published: 30 August 2014

\section{References}

Azor M, Gené J, Cano J, Manikandan P, Venkatapathy N, Guarro J (2009) Less-frequent Fusarium species of clinical interest: correlation between morphological and molecular identification and antifungal susceptibility. J Clin Microbiol 47:1463-1468

Bodey GP, Boktour M, Mays S, Duvic M, Kontoyiannis D, Hachem R, Raad I (2002) Skin lesions associated with Fusarium infection. J Am Acad Dermatol 47:659-666

Bose P, Parekh HD, Holter JL, Greenfield RA (2011) Disseminated fusariosis occurring in two patients despite posaconazole prophylaxis. J Clin Microbiol 49:1674-1675

Bourgeois GP, Cafardi JA, Sellheyer K, Andea AA (2010) Disseminated Fusarium originating from toenail paronychia in a neutropenic patient. Cutis 85:191-194

Busemann C, Krüger W, Schwesinger G, Kallinich B, Schröder G, Abel P, Kiefer T, Neumann T, Dölken G (2009) Myocardial and aortal involvement in a case of disseminated infection with Fusarium solani after allogeneic stem cell transplantation: report of a case. Mycoses 52:372-376

Calcaterra D, Karam K, Suzuki Y (2013) Computed tomography findings in a patient with fungal aortitis: acute aortic syndrome secondary to fusariosis. Interact Cardiovasc Thorac Surg 17:171-172

Cho CT, Vats TS, Lowman JT, Brandsberg JW, Tosh FE (1973) Fusarium solani infection during treatment for acute leukemia. J Pediatr 83:1028-1031

CLSI (2008) Reference Method for Broth Dilution Antifungal Susceptibility Testing of Filamentous Fungi; Approved Standard-Second Edition. CLSI document M38-A2. Clinical and Laboratory Standards Institute, Wayne, PA

Cooke NS, Feighery C, Armstrong DK, Walsh M, Dempsey S (2009) Cutaneous Fusarium solani infection in childhood acute lymphoblastic leukaemia. Clin Exp Dermatol 34:e117-e119

De Pinho DB, Fernandes LL, Carvalho Barreiros Mda G, Quintella LP, Sodré CT, Ramos-E-Silva M (2012) Disseminated fusariosis in a bone marrow transplant patient. J Clin Aesthet Dermatol 5:40-42

Durand-Joly I, Alfandari S, Benchikh Z, Rodrigue M, Espinel-Ingroff A, Catteau B, Cordevant C, Camus D, Dei-Cas E, Bauters F, Delhaes L, De Botton S (2003) Successful outcome of disseminated Fusarium infection with skin localization treated with voriconazole and amphotericin B-lipid complex in a patient with acute leukemia. J Clin Microbiol 41:4898-4900

Fanci R, Pini G, Bartolesi AM, Pecile P (2013) Refractory disseminated fusariosis by Fusarium verticillioides in a patient with acute myeloid leukaemia relapsed after allogeneic hematopoietic stem cell transplantation: a case report and literature review. Rev Iberoam Micol 30:51-53

Ferrari MD, Resende MR, Sakai K, Muraosa Y, Lyra L, Gonoi T, Mikami Y, Tominaga K, Kamei K, Schreiber AZ, Trabasso P, Moretti ML (2013) Accurate visual 
DNA-microarray for the molecular identification of non-albicans Candida species isolated from candidemia episodes. J Clin Microbiol 51:3826-3829

Homa M, Shobana CS, Singh YR, Manikandan P, Selvam KP, Kredics L, Narendran V, Vágvölgyi C, Galgóczy L (2013) Fusarium keratitis in South India: causative agents, their antifungal susceptibilities and a rapid identification method for the Fusarium solani species complex. Mycoses 56:501-511

Galimberti R, Torre AC, Baztán MC, Rodriguez-Chiappetta F (2012) Emerging systemic fungal infections. Clin Dermatol 30:633-650

Gupta AK, Baran R, Summerbell RC (2000) Fusarium infections of the skin. Curr Opin Infect Dis 13:121-128

Guzman-Cottrill JA, Zheng X, Chadwick EG (2004) Fusarium solani endocarditis successfully treated with liposomal amphotericin $B$ and voriconazole. Pediatr Infect Dis J 23:1059-1061

Ho DY, Lee JD, Rosso F, Montoya JG (2007) Treating disseminated fusariosis: amphotericin B, voriconazole or both? Mycoses 50:227-231

Inano S, Kimura M, lida J, Arima N (2013) Combination therapy of voriconazole and terbinafine for disseminated fusariosis: case report and literature review. $J$ Infect Chemother 19:1173-1180

Jureen R, Koh TH, Wang G, Chai LY, Tan AL, Chai T, Wong YW, Tambyah PA, Beuerman R, Tan D (2008) Use of multiple methods for genotyping Fusarium during an outbreak of contact lens associated fungal keratitis in Singapore. BMC infectious Disease 8:92

Liu JY, Chen WT, Ko BS, Yao M, Hsueh PR, Hsiao CH, Kuo YM, Chen YC (2011) Combination antifungal therapy for disseminated fusariosis in immunocompromised patients: a case report and literature review. Med Mycol 49:872-878

Makimura K, Oguri T, Mikami Y, Kume H, Hanazawa R, Abe M, Ikeda R, Shinoda T (2005) Multicenter evaluation of commercial frozen plates for microdilution broth antifungal susceptibility testing of yeasts and comparison of MIC limits recommended in NCCLS M27-A2. Microbiol Immunol 49:97-106

Martino P, Gastaldi R, Raccah R, Girmenia C (1994) Clinical patterns of Fusarium infections in immunocompromised patients. J Infect 1:7-15

Melcher GP, McGough DA, Fothergill AW, Norris C, Rinaldi MG (1993) Disseminated hyalohyphomycosis caused by a novel human pathogen, $F$. napiforme. J Clin Microbiol 31:1461-1467

Moreira-Oliveira MS, Mikami Y, Miyaji M, Imai T, Schreiber AZ, Moretti ML (2005) Diagnosis of candidemia by polymerase chain reaction and blood culture: prospective study in a high-risk population and identification of variables associated with development of candidemia. Eur J Clin Microbiol Infect Dis 24:721-726

Neuburger S, Massenkeil G, Seibold M, Lutz C, Tamm I, le Coutre P, Graf B, Doerken B, Arnold R (2008) Successful salvage treatment of disseminated cutaneous fusariosis with liposomal amphotericin B and terbinafine after allogeneic stem cell transplantation. Transpl Infect Dis 10:290-293

Nucci M, Anaissie E (2007) Fusarium infections in immunocompromised patients. Clin Microbiol Rev 20:695-704

Nucci M, Garnica M, Gloria AB, Lehugeur DS, Dias VC, Palma LC, Cappellano P, Fertrin KY, Carlesse F, Simões B, Bergamasco MD, Cunha CA, Seber A, Ribeiro MP, Queiroz-Telles F, Lee ML, Chauffaille ML, Silla L, de Souza CA, Colombo AL (2013) Invasive fungal diseases in haematopoietic cell transplant recipients and in patients with acute myeloid leukaemia or myelodysplasia in Brazil. Clin Microbiol Infect 19:745-751

Pereira GH, de Angelis DA, Brasil RA, dos Anjos Martins M, De Matos Castro e Silva D, Szeszs MW, de Souza Carvalho Melhem M (2013) Disseminated amphotericin-resistant fusariosis in acute leukemia patients: report of two cases. Mycopathologia 175:107-114

Rodriguez CA, Luján-Zilbermann J, Woodard P, Andreansky M, Adderson EE (2003) Successful treatment of disseminated fusariosis. Bone Marrow Transplant 31:411-412

Scheel CM, Hurst SF, Barreiros G, Akiti T, Nucci M, Balajee SA (2013) Molecular analyses of Fusarium isolates recovered from a cluster of invasive mold infections in a Brazilian hospital. BMC Infect Dis 13:49

Rothe A, Seibold M, Hoppe T, Seifert H, Engert A, Caspar C, Karthaus M, Fätkenheuer G, Bethe U, Tintelnot K, Cornely OA (2004) Combination therapy of disseminated Fusarium oxysporum infection with terbinafine and amphotericin B. Ann Hematol 83:394-397

Sakai K, Trabasso P, Moretti LM, Mikami Y, Kamei K, Gonoi T (2014) Identification of fungal pathogens by visible microarray 4 system in combination with isothermal gene amplification. Mycophatologia 178:11-26

Sekeroglu TH, Erdem E, Yagmur M, Gumral R, Ersoz R, Ilkit M, Harbiyeli II (2012) Successful medical management of recalcitrant Fusarium solani keratitis: molecular identification and susceptibility patterns. Mycopathologia 174:233-237

Slavin MA, Chakrabarti A (2012) Opportunistic fungal infections in the Asia-Pacific region. Med Mycol 50:18-25

Teixeira ABA, Moretti ML, Trabasso P, Nowakonshi AV, Aoki FH, Vigorito AC, Miyaji M, Nishimura K, Taguchi H, Schreiber AZ (2005) Evaluation of Fusarium solani hyphae and conidia susceptibility to amphotericin B and itraconazole: study of a clinical case. Mycophatologia 116:291-296

Tezcan G, Ozhak-Baysan B, Alastruey-Izquierdo A, Ogunc D, Ongut G, Yildiran ST, Hazar V, Cuenca-Estrella M, Rodriguez-Tudela JL (2009) Disseminated fusariosis caused by Fusarium verticillioides in an acute lymphoblastic leukemia patient after allogeneic hematopoietic stem cell transplantation. J Clin Microbiol 47:278-281

Tortorano AM, Prigitano A, Dho G, Esposto MC, Gianni C, Grancini A, Ossi C, Viviani MA (2008) Species distribution and in vitro antifungal susceptibility patterns of 75 clinical isolates of Fusarium spp. from Northern Italy. Antimicrob Agents Chemother 52:2683-2685

Verweij PE, Brandt ME (2007) Aspergillus, Fusarium, and Other Opportunistic Moniliaceous Fungi. In: Murray PR, Baron EJ, Jorgensen JH, Landry ML (eds) Pfaller MA Manual of clinical microbiology, 9th edn. ASM Press, Washington, DC Xie L, Zhai H, Zhao J, Sun S, Shi W, Dong X (2008) Antifungal susceptibility for common pathogens of fungal keratitis in Shandong Province, China. Am J Ophthalmol 146:260-265

\section{doi:10.1186/2193-1801-3-492}

Cite this article as: de Souza et al:: Fusarium napiforme systemic infection: case report with molecular characterization and antifungal susceptibility tests. SpringerPlus 2014 3:492.

\section{Submit your manuscript to a SpringerOpen ${ }^{\odot}$ journal and benefit from:}

- Convenient online submission

$\checkmark$ Rigorous peer review

- Immediate publication on acceptance

- Open access: articles freely available online

- High visibility within the field

- Retaining the copyright to your article

Submit your next manuscript at $>$ springeropen.com 